\title{
L'APRIORISME DES AUTRICHIENS
}

\author{
Maurice LAGUEUX ${ }^{1}$
}

\begin{abstract}
Résumé
L'apriorisme radical prôné par Mises et Rothbard a été maintes fois dénoncé avec véhémence. Sans chercher le moindrement à réhabiliter cette thèse intempérante, on s'intéressera à l'intuition d'origine kantienne qui est à sa racine. En prenant pour exemple la "loi" de l'utilité marginale décroissante, on élaborera une expérience imaginaire qui permettra de comparer l'attachement des économistes envers ce genre de loi à celui que, selon Kuhn, les physiciens éprouvent à l'égard de leurs théories. Cette discussion permettra alors de préciser et d'illustrer en quoi consiste la condition d'intelligibilité dont on a cru indûment pouvoir dériver une thèse résolument aprioriste.
\end{abstract}

\begin{abstract}
The radical apriorism of Mises and Rothbard has frequently been violently criticized. Without attempting to rehabilitate this excessive thesis, the paper will revisit the intuition, originating in Kant, from which apriorism was developed. Taking as an example the "law" of diminishing marginal utility, it will propose an imaginary experiment that will allow us to compare economists' loyalty towards this kind of law to the loyalty that, according to Kuhn, physicists have to their theories. Through this discussion, it will specify and illustrate the nature of the condition of intelligibility from which a straightforwardly apriorist thesis has been incorrectly derived.
\end{abstract}

Classification JEL : B41, B53

Il n'y a pas si longtemps, la notion même d'apriorisme était de celles qui suscitaient les plus hostiles réactions dans les milieux académiques. Depuis la parution du célèbre article de Milton Friedman (1953), il était de bon ton de valoriser plutôt une économie qualifiée de positive ou d'empirique. Déjà dénoncée sévèrement, quoique indirectement, par Félix Kaufmann (1937), l'application d'une thèse aprio-

1. Université de Montréal. L'auteur tient à remercier Stéphane D'Amour, Richard Ebeling, JeanJacques Gislain, Daniel Hausman, Roger Koppl, Gérald Lafleur, Philippe Mongin, Robert Nadeau, François Tournier et un évaluateur des CEP dont les commentaires, à une étape ou l'autre de la préparation de ce travail, lui ont été particulièrement utiles, même si, bien entendu, aucun d'eux ne devrait être présumé en accord avec ses conclusions. L'aide financière du CRSH (Ottawa) et du Fonds FCAR (Québec) a été aussi fort appréciée. 
riste à l'économie devait constituer une proie relativement facile pour les quelques économistes comme T.W. Hutchison (1956), J.J. Klant (1984, ch. 2) et, en France, Claude Meidinger (1978) qui, au cours du dernier demi-siècle, ont daigné y porter attention. Cette doctrine suscitait même une désapprobation si générale que, en commentant un article (Caldwell, 1984) que Bruce Caldwell avait consacré à l'examen des débats autour de cette question, Eugen Rotwein (1986) pouvait le plus sérieusement du monde reprocher à son auteur d'avoir "flirté" avec l'apriorisme. Dans ce contexte, on comprend combien la théorie économique autrichienne a pu souffrir d'avoir été souvent associée à ce type d'approche épistémologique. Même si tous les Autrichiens sont loin d'avoir endossé l'apriorisme radical d'un Mises ou d'un Rothbard, le fait que la plupart d'entre eux se soient montrés plutôt réticents face à l'empirisme inconditionnel des ennemis jurés de l'apriorisme a beaucoup contribué à accréditer l'idée voulant qu'une forme plus ou moins explicite d'apriorisme constituait une composante honteuse de la méthodologie autrichienne.

De nos jours, l'apriorisme n'a pas encore obtenu droit de cité parmi les thèses épistémologiques jugées respectables, mais à la faveur de l'insatisfaction assez généralisée qu'inspirent désormais l'empirisme et le positivisme qui triomphaient jadis, on a pu au moins porter sur la question un regard plus serein. C'est ainsi que les articles de Barry Smith (1996, voir aussi 1986) et de Stephen Parsons (1997, voir aussi 1990) ont contribué à conférer une certaine crédibilité à une forme d'apriorisme dont il faut bien reconnaître cependant qu'elle a peu à voir avec l'apriorisme radical d'un Mises. L'ingénieux argument que Parsons (1997) dérive d'une relecture de Mises de manière à mieux asseoir les vues de Kant sur la causalité ne permet guère, en effet, de comprendre pourquoi Mises voit dans la théorie économique un exemple privilégié de connaissance a priori. De même, les nombreuses propositions que Smith (1996) présente comme a priori tout en reconnaissant qu'elles peuvent être fondées sur une "expérience intuitive" et qu'elles pourraient fort bien se révéler "fausses", ne contribuent que bien indirectement à revaloriser les intuitions plus ou moins aprioristes qui ont été associées à la pensée autrichienne. Mises et Rothbard, en tout cas, n'hésitaient pas à présenter la théorie économique elle-même comme une connaissance a priori et de ce fait infaillible et indépendante de toute forme d'expérience.

Or sans chercher ici à défendre une forme aussi intempérante d'apriorisme, je voudrais faire voir en quoi l'apriorisme des Autrichiens met néanmoins l'accent sur une dimension incontournable de la théorie économique. Qu'on se rassure, il ne saurait être question ici d'entériner les conclusions, parfaitement abusives à mon sens, auxquelles l'apriorisme est le plus souvent associé. Il parait, en effet, bien inacceptable d'opposer apriorisme et empirisme comme s'il s'agissait de deux voies différentes par lesquelles on pourrait acquérir des connaissances économiques valables, l'une qui exigerait constamment de recourir à l'expérience et l'autre qui autoriserait allègrement à se dispenser d'y faire appel. Il faut reconnaître que bien des propos de Mises et de Rothbard semblent légitimer, ou presque, une telle interprétation. Mises 
nous parle, par exemple, de lois auxquelles il faut apparemment reconnaître une dignité à tout le moins comparable à celles des physiciens, mais qui seraient pourtant "dérivées a priori" et ne toléreraient "aucune exception" puisqu'elles relèveraient d'une "théorie aprioriste et universellement valable" (1976, pp., 197 et xxvii). Quant à Rothbard, s'il admet que l'axiome d'où dériveraient de telles connaissances pourrait reposer sur une "expérience interne", il assure que ce type d'expérience, qui permettrait de dégager une "loi de la réalité" absolument irréfutable, n'a rien à voir avec l'expérience qu'invoque l'empirisme (1957, p. 317-318). Or, pour des raisons qui rejoignent celles qui ont inspiré les études critiques mentionnées plus haut, il ne sera pas question ici de laisser entendre que des lois qui régiraient un pan entier de l'activité humaine puissent être reconnues comme telles sans qu'il y ait lieu de les soumettre à l'épreuve de l'expérience ${ }^{2}$.

Ce qu'il s'agira, par contre, de mettre en relief, c'est une intuition fondamentale qui me paraît présente à des degrés divers chez la plupart des Autrichiens et qui se trouve exprimée de façon en quelque sorte exacerbée dans l'apriorisme de Mises. Cette intuition est avant tout celle qui veut que les sciences qui s'intéressent aux actions humaines diffèrent des sciences naturelles en ceci qu'une action doit être comprise et qu'elle ne peut l'être en l'absence de certaines conditions qui tiennent à sa nature même et qu'on désignera ici du nom de conditions d'intelligibilité — en mettant l'accent sur une dimension que Lachmann et Kirzner, mieux que Mises, ont su mettre en relief ${ }^{3}$. Ces conditions de possibilité d'une authentique compréhension des actions humaines précèdent forcément toute expérience, mais elles ne devraient pas pour autant être confondues avec la connaissance qu'elles rendent possible. Toutefois, comme elles concernent la structure même de l'action humaine, la tentation est grande d'y voir une sorte de paradigme de cette connaissance elle-même et c'est de cette tentation qu'est né l'apriorisme de Mises. On objectera cependant aux accents manifestement kantiens de cette façon de voir que si Mises s'est volontiers réclamé de Kant, il n'en a pas forcément adopté les thèses épistémologiques, puisqu'il n'hésite pas à soutenir que si la science économique et l'ensemble de la praxéologie, tout comme les mathématiques d'ailleurs, s'imposent a priori, c'est que leur contenu serait purement analytique (Mises, 1985, p. 42).

Or, sur ce point, on se heurte chez Mises à une incohérence qui se répercute dans l'ensemble de son œuvre. Bien qu'il qualifie volontiers sa praxéologie de démarche analytique, sa façon de la présenter en fait une connaissance qui, si elle est vraiment a priori, ne saurait être que synthétique a priori ${ }^{4}$. Dans The Ultimate

2. Dans des travaux qui ne sont pas que critiques à l'égard de l'apriorisme de Mises, Barrotta (1996 et 1998), soutient également que cet apriorisme ne saurait autoriser la dérivation de la théorie économique que Mises croyait pouvoir en tirer.

3. Voir, par exemple, Lachmann 1969, p. 92 et Kirzner, 1976, pp. 41 et 44-46.

4. On sait que Kant qualifie d'analytique un jugement de type "A est B" dont le prédicat B appartient littéralement au sujet $\mathrm{A}$. Les jugements analytiques, dont les définitions constituent des exemples- 
Foundation of Economic Science, par exemple, il reproche au positivisme logique de rejeter les propositions synthétiques a priori $(1978$, pp. 5) et de conclure, du seul fait de l'existence des géométries non-euclidiennes, que la géométrie repose sur des axiomes arbitraires (1978, pp. 5 et 13-14). Ainsi, tout analytique qu'elle soit censée être, la géométrie "nous serait imposée par le monde dans lequel nous vivons", au même titre d'ailleurs que la logique, l'ensemble des mathématiques et la praxéologie, sans quoi "aucune connaissance ne serait accessible à l'homme" (1978, p.14). En ce qui a trait aux catégories logiques fondamentales, Mises est encore plus explicite: "l'esprit humain est totalement incapable d'imaginer des catégories logiques autres que celles-là" (1985, p. 39). Dans l'Action humaine, il assure que "les sciences aprioristes - logique, mathématiques et praxéologie - tendent à un savoir inconditionnellement valable pour tous les êtres dotés de la structure logique de l'esprit humain" (1985, p. 62; voir aussi p. 36 et 1978, p. 16). Pourtant, il avait mis sa praxéologie à l'abri de toute objection qui aurait pu prendre appui sur une conception autre de la nature des mathématiques en assurant que ce qui intéresse ces dernières n'a rien à voir avec la praxéologie, puisque celle-ci repose sur une connaissance "évidente en elle-même" du "fait qu'il existe quelque chose qui cherche consciemment à réaliser des fins" (1978, pp. 5-6; voir aussi p.18). Et à qui demanderait d'où nous vient cette connaissance, Mises pouvait toujours répondre, comme il le faisait déjà dans l'Action humaine, que "la connaissance de l'essence de l'agir humain" est "nôtre parce que nous sommes des hommes" (1985, p. 69). Il va même jusqu'à invoquer le fait que la structure de l'esprit humain résulte d'un processus de sélection naturelle pour soutenir que les catégories a priori qui en dérivent sont porteuses d'une "certaine information ayant trait à la réalité de l'Univers" $(1978,16)$. Avec les a priori, ajoutera-t-il dans The Ultimate Foundation, "nous avons affaire aux outils mentaux qui nous rendent aptes à expérimenter, à apprendre, à connaître et à agir" (1978, pp. 18-19). Sans doute pourra-t-on contester la possibilité même de disposer a priori de tels outils et d'une telle connaissance "imposée par le monde", mais on conviendra que, s'ils existaient, ces outils auraient un caractère franchement synthétique, ne serait-ce que du fait qu'ils nous informent manifestement sur la nature des hommes qui vivent dans le monde.

Alors pourquoi Mises tenait-il tant à affirmer le caractère analytique de la science économique? Sans doute parce qu'il était fasciné par l'idée d'une science qui

types, ne peuvent donc nous apprendre quoi que ce soit sur l'état du monde, puisqu'ils ne font que clarifier le contenu du terme qu'ils prennent pour sujet. Du coup, la science économique, qui prétend nous éclairer sur la façon dont fonctionne un secteur du monde réel, ne peut reposer uniquement sur des jugements analytiques. Par contraste, Kant qualifie de synthétique un jugement dont le prédicat n'appartient en rien au sujet lui-même. Un tel jugement peut donc nous apprendre que, dans le monde réel, un prédicat donné est effectivement attribuable au sujet de ce jugement. Si la science économique porte sur le monde réel, elle doit donc être constituée pour l'essentiel de jugements synthétiques; aussi, soutenir qu'elle est néanmoins a priori, c'est faire appel à des jugements synthétiques qui peuvent être dits synthétiques a priori. D'où, quant on a affaire à une approche qui se réclame de Kant, l'importance de la question de savoir s'il est acceptable de se référer à de tels jugements. 
se déduit more geometrico, ce qui correspond, explique-t-il, au "grand idéal des chercheurs de vérité" $(1978$, p. 5). Mises soupçonnait bien qu'une théorie économique ne pouvait pas plus être déduite de catégories synthétiques a priori que la physique classique pouvait être déduite des a priori kantiens. Une théorie économique de caractère purement analytique pouvait donc mieux satisfaire son idéal de "chercheur de vérité" pour peu qu'elle puisse être appliquée au monde réel là où il y a correspondance entre ce monde réel et les axiomes de cette théorie. Mais si Mises s'en était tenu rigoureusement à cette façon de voir, il n'aurait pu se dire aprioriste. Un arpenteur qui constate qu'un terrain est triangulaire et qui en conclut que la somme de ses angles est égale à deux droits n'est pas aprioriste pour autant; il utilise simplement une connaissance de caractère analytique pour tirer une conclusion à propos d'un objet qu'il a mesuré empiriquement. Or quand Mises prétend que la praxéologie est purement analytique, il se prive de la possibilité de faire plus que cet arpenteur. Il ne serait pas aprioriste, en effet, s'il se contentait de soutenir que si l'on pouvait établir empiriquement que les hommes agissent conformément à ce qui est postulé par son modèle praxéologique, on pourrait légitimement prendre appui sur cette praxéologie analytique pour déduire de ce qui a été ainsi établi diverses conclusions d'intérêt économique. Mais il est clair, et on l'a vu plus haut, que l'apriorisme de Mises va beaucoup plus loin et qu'il ne se réduit pas à proposer, pour utilisation en temps utile, une sorte de grammaire (analytique) des concepts économiques.

\section{Connaissance a priori ou condition d'intelligibilité}

On peut donc, sans crainte de trahir l'intuition qui est à la racine de l'apriorisme des Autrichiens oublier cette maladroite référence au caractère purement analytique de l'analyse économique et s'interroger plutôt, dans un esprit forcément plus kantien, sur les conditions de possibilité a priori de la connaissance économique. Kant, on le sait, s'est intéressé avant tout à la seule science qui, à son époque, lui paraissait incontestablement valable, soit la physique de Newton. Or selon Mises et certains de ses collègues autrichiens, il convient d'abord de mettre en relief la dualité fondamentale entre les sciences physiques et les sciences de l'action. Mises défend la chose avec beaucoup de conviction dans l'introduction de Theory and History, dont le tout premier paragraphe souligne l'importance du "dualisme méthodologique" (1985a, p. 1). Rothbard, pour sa part, amorce son intervention en faveur de l'"apriorisme extrême" en assurant que le cœur du débat se trouve dans la confusion entre sciences physiques et sciences de l'action humaine (1957, p. 315). Or pour Mises, on l'a vu, le privilège de ces dernières tient au fait que "nous sommes des hommes", en conséquence de quoi l'activité humaine peut être comprise directement et ainsi rendue intelligible en un sens qui ne saurait avoir d'équivalent quand on a affaire au monde physique. Il est assez clair que cette idée, qui avait pris forme au sein de la tradition autrichienne, n'était pas étrangère à la notion de Verstehen 
développée par Dilthey, Weber et d'autres penseurs allemands ${ }^{5}$, mais ce qui importe ici, c'est qu'elle est invoquée sous une forme ou une autre dès que l'on entend dégager les conditions de possibilité qui seraient propres aux sciences humaines.

On peut illustrer à l'aide de l'exemple suivant en quoi cette façon de voir permet de mettre en relief une différence importante entre sciences physiques et sciences humaines. Si, sans la moindre connaissance de l'économie, j'observe qu'un agriculteur qui s'efforce de vendre ses pommes au marché les cède de préférence à celui de ses clients potentiels qui lui offre en retour la somme la plus élevée, je comprends immédiatement son comportement et cette compréhension immédiate tient beaucoup au fait qu'un tel comportement paraît dicté par un choix rationnel relevant du type de rationalité qui guide normalement mon propre comportement. Si, par contre, je le voyais céder plus volontiers ses pommes à celui de ses clients potentiels qui lui offre la plus petite somme, j'éprouverais une frustrante sensation d'incompréhension et j'exigerais une explication supplémentaire (désir altruiste d'aider les clients les moins fortunés, doute sérieux sur la qualité de son produit, vif plaisir éprouvé à surprendre les badauds, etc.), alors qu'aucune explication de ce genre ne serait requise si je le voyais agir de la première façon. À défaut d'une telle explication, je serais fort tenté de conclure que l'individu a perdu la raison et me désolerais de me heurter à un comportement nettement irrationnel dont je ne comprends pas la logique. Par contre, si je constate qu'un corps se dilate systématiquement à la chaleur, je cherche certes une explication; mais je le ferais, exactement au même titre, si, sans la moindre connaissance de la physique, je constatais qu'il se contracte. Je n'aurais pas alors de raison particulière d'être stupéfait, car il n'y aurait évidemment aucun sens à soutenir qu'une contraction causée par la chaleur serait quelque chose d'incompréhensible et à appuyer cette allégation sur une prétendue perception immédiate de la dilatation de mon propre corps à la chaleur.

Sans doute, cherche-t-on à se rendre intelligibles les phénomènes naturels tout autant que les actions humaines, mais les premiers seront réputés intelligibles s'ils peuvent être perçus comme de simples cas, parmi tant d'autres, d'un phénomène régi par une loi générale, sans que nous ayons d'exigences a priori quant à la nature de celle-ci. Par contre, les actions humaines ne seront réputées intelligibles que s'il est manifeste qu'elles se conforment au principe de rationalité ou, en d'autres mots, si elles laissent percevoir la logique au nom de laquelle les moyens qui y sont mis en œuvre sont commandés par un objectif quelconque. Bien sûr, la connaissance des qualités propres aux pommes et au papier-monnaie n'est pas plus une connaissance a priori que ne l'est celle ayant trait à la dilatation des corps; la seule chose que l'on peut qualifier d'a priori, c'est l'exigence d'une relation qui puisse être qualifiée de

5. Sur les parentés entre la tradition autrichienne et celle qui est associée au Verstehen, voir Lachmann, 1969, p. 94 et Alter, 1990, pp. 320-332. 
rationnelle. Telle est la condition d'intelligibilité qu'exige a priori la compréhension des actions humaines. Ou bien une telle condition est satisfaite, ou bien il faut avouer qu'on ne comprend rien à l'action qu'il s'agit d'expliquer. Cette exigence peut donc être dite a priori, mais, on le voit, le fait de l'invoquer ne signifie pas que nous disposons d'une connaissance supplémentaire, mais bien plutôt que nous sommes forcés d'admettre que notre compréhension des actions humaines ne peut se passer de cette rationalité minimale. À défaut de celle-ci, nous sommes forcés d'avouer que nous n'y comprenons littéralement rien. C'est ce modeste constat d'une différence significative entre ce que requiert la compréhension des phénomènes observés dans l'un et l'autre type de science qui, à mon sens, est à l'origine de l'apriorisme indûment radicalisé et généralisé par Mises. Il est vrai que Rothbard n'accepterait pas cette façon de voir puisqu'il soutient que la rationalité ne joue aucun rôle dans la pensée de Mises qui ne recourrait jamais à ce concept (Rothbard, 1957, p. 317). La vérité, c'est que Mises n'a aucune raison d'utiliser le concept de rationalité puisque, soutient-il, "[1]'agir humain est nécessairement toujours rationnel. Le terme 'action rationnelle' est ainsi pléonasmatique et doit être évité comme tel" $(1985$, p. 21 ; voir aussi 1944, p. 544). Tout au plus faut-il reconnaître que Mises ne fait aucune place à la conception de la rationalité qu'invoquent maints économistes selon qui, pour être dit rationnel, un comportement doit maximiser une variable prédéterminée, puisque chez lui la rationalité de l'action correspond avant tout à l'idée voulant que toute action soit présumée nécessairement orientée vers un but quelconque.

Quoi qu'il en soit de ce débat quelque peu sémantique, on peut illustrer ce qu'il en est de "l'apriorisme" qui caractérise la façon de voir exposée ci-dessus, à l'aide de l'une de ces prétendues lois économiques que Mises et ses disciples prétendent connaître a priori, soit la "loi" voulant que l'utilité marginale soit décroissante (Mises, 1985, ch. VII). À première vue, cette prétendue loi semble comporter de nombreuses exceptions: qu'on pense à l'alcoolique prêt à payer plus pour le second verre que pour le premier, ou, pour reprendre un exemple cité par Rothbard lui-même, au cuisinier qui éprouve plus de satisfaction à mettre la main sur le quatrième des quatre œufs requis par une recette que par l'acquisition du premier de ces œufs (Rothbard, 1962, p. 64). De telles exceptions ne pouvaient toutefois perturber un aprioriste comme Rothbard, non plus, à vrai dire, que la plupart des économistes. Après tout, ce que ce cuisinier cherchait à se procurer pour satisfaire son désir de cuisiner n'était pas un œuf mais plutôt un quarteron d'œufs. Si ce cuisinier trouvait ensuite un deuxième, puis un troisième ensemble de quatre œufs, il en éprouverait sans doute une satisfaction de moins en moins grande. Quant à l'alcoolique, il ne saurait être considéré par l'économiste comme un consommateur suffisamment représentatif puisque ses goûts changent en cours de route; n'était l'accentuation physiologique de son besoin d'alcool occasionnée par la déglutition des premiers verres, son désir de satisfaire une soif donnée diminuerait avec chaque verre. Pourtant, si l'on y voit un moyen de sauver à tout prix une loi qui est censée s'imposer à toutes les actions humaines et être comparable à la loi de la chute des corps, ces considérations ad hoc paraitront fort peu convaincantes. En effet, si une loi physique comme 
celle de la chute des corps, semble connaître une exception quand une bulle s'envole vers le ciel, une telle "exception" est vite expliquée à l'aide d'autres lois, lesquelles sont appuyées sur des bases empiriques autres, et non pas de façon purement ad hoc comme l'a été ci-dessus la prétendue loi de l'utilité marginale décroissante.

Quoi qu'il en soit, si tant d'économistes continuent de penser que le cuisinier éprouverait sans doute une satisfaction de moins en moins grande avec l'arrivée de nouveaux quarterons d'œufs et que le buveur à physiologie gustative constante éprouverait de moins en moins de plaisir à boire un nouveau verre, c'est que leur conviction repose sur des bases différentes, car on a affaire là non pas à une loi comparable à celle de la chute des corps, mais bien plutôt à une condition d'intelligibilité. En l'absence d'une telle condition, on ne pourrait rien comprendre au comportement économique des êtres humains, lequel par contre nous parait généralement compréhensible dès qu'on la tient pour acquise. La question est donc de savoir ce qui fonde la conviction - partagée à vrai dire par nombre d'économistes qui ne se considèrent pas aprioristes pour autant - voulant que l'utilité marginale ne puisse être que décroissante ${ }^{6}$. D'aucuns invoqueront les études psychologiques qui analysent empiriquement le phénomène que constitue la satiété, mais les économistes ont trop cherché depuis un siècle à se libérer de toute dépendance à l'égard de la psychologie pour que l'on puisse penser que leur conviction à l'égard du caractère décroissant de l'utilité marginale repose essentiellement sur les résultats des émules de Fechner. Dans la tradition autrichienne, on le sait, on a plutôt invoqué un argument intuitif du type suivant. Puisqu'une action est orientée vers la satisfaction d'un besoin, ce besoin est jugé par l'agent plus important que les autres besoins qu'il aurait pu satisfaire s'il avait plutôt choisi d'agir autrement. Conséquemment, quand un agent acquiert une première unité d'un bien donné, il le consacre à la satisfaction au moins partielle du besoin qui lui paraît le plus important ou le plus urgent parmi ceux que ce bien peut satisfaire. S'il en est ainsi - à moins que ne soient modifiés en cours de route, ou ce besoin lui-même, ou les goûts de celui qui le poursuit, ou la nature des biens qui sont susceptibles de le satisfaire,

6. Sans doute, bien des économistes contemporains feront observer qu'ils ne se préoccupent guère de savoir si l'utilité marginale est décroissante ou pas, puisqu'il leur suffit d'admettre que le taux marginal de substitution entre les biens est décroissant. Toutefois, comme Hicks et Allen (1934) le faisaient voir clairement, ce taux marginal (décroissant) de substitution entre deux biens a pu remplacer l'utilité marginale (décroissante) d'un bien donné — notion invoquée par Jevons et ses successeurs — parce qu'il n'est rien d'autre qu'un rapport entre utilités marginales. En fait, le caractère décroissant de ces deux entités correspond à deux façons équivalentes d'intégrer le principe de rationalité à l'analyse économique, car la décroissance du taux marginal de substitution tient au fait que la variation du nombre d'unités d'un bien exigé en échange d'un autre dépend de la variation de l'utilité que procure ce dernier à mesure que la quantité qui en est détenue augmente ou diminue. Pour une brève discussion de cette question, voir Lagueux (2004), principalement en p. 36. Dans le présent article, il n'est question que de la décroissance de l'utilité marginale, à la fois parce que la discussion principale s'en trouve grandement facilitée et clarifiée et parce qu'il s'agit là d'un des points assez rares où l'approche des Autrichiens rejoint celle adoptée par Jevons et, quoique indirectement comme on vient de le voir, celle de l'économie contemporaine. 
ou quelque autre paramètre essentiel —, la seconde unité acquise du même bien ne pourra qu'apporter une contribution de moindre importance que la première (ou, tout au plus, d'importance égale) à la satisfaction d'un besoin quelconque, et ainsi de suite pour les unités subséquentes. En effet, le besoin que la première unité a contribué à satisfaire, l'a été, par hypothèse, dans ce qu'il avait de plus urgent et tous les autres besoins ont, par hypothèse également, été jugés moins importants lors de l'usage de cette première unité. Compris de cette façon, le caractère décroissant de l'utilité marginale est donc bien moins une loi qu'une condition d'intelligibilité sans laquelle les choix économiques deviendraient littéralement inintelligibles parce que dépourvus de toute logique.

Mais peut-on montrer qu'une telle "condition d'intelligibilité" s'impose vraiment? La chose paraît difficile, mais peut-être peut-on mettre en relief l'embarras dans lequel se verraient plongés les économistes s'ils s'avisaient de ne pas respecter une telle condition. Le seul fait de constater un tel embarras conférerait, à tout le moins, un peu de crédibilité à l'idée voulant qu'une telle condition ait quelque chose d'incontournable. De fait, s'ils étaient obligés de choisir entre deux théories également satisfaisantes du point de vue de leur pouvoir de prédiction, mais dont l'une invoquerait un principe d'utilité marginale décroissante et l'autre un principe d'utilité marginale croissante, il est probable que les économistes ne se montreraient pas indifférents et qu'ils seraient fortement enclins à opter pour la première. Si tel est bien le cas, il paraît raisonnable de penser que cette préférence épistémologique présumée tiendrait à la conviction que l'abandon du principe d'utilité marginale décroissante aurait pour conséquence de rendre l'action humaine carrément inintelligible. Bien sûr, rien de tout cela n'autoriserait à conclure au bien-fondé de l'apriorisme, mais on pourrait néanmoins en conclure que, aux yeux des économistes, le sacrifice de l'intelligibilité rendue possible par le recours au principe d'utilité marginale décroissante serait un prix à payer trop élevé pour que l'on puisse admettre qu'un tel sacrifice soit vraiment exigé par la pratique scientifique. Peut-être comprendrait-on mieux alors que certains économistes autrichiens aient pu être amenés à voir dans cette condition d'intelligibilité, une condition qui s'impose a priori et, de là, à en faire, à tort à mon sens, le point de départ d'une théorie économique a priori.

Toutefois, même si la préférence épistémologique des économistes pour l'utilité marginale décroissante était incontestablement établie, on pourrait penser qu'il s'agit là de quelque chose d'assez analogue à l'attachement que le physicien manifeste à l'endroit d'un "paradigme" qui l'a bien servi, pour employer le langage de Thomas Kuhn. Or celui-ci a bien montré que même si les physiciens s'attachent à leurs théories pour des raisons fort compréhensibles, leurs successeurs, à tout le moins, seront peu à peu conduits à abandonner celles-ci au profit de théories basées sur des postulats opposés si ces derniers se révèlent empiriquement plus fructueux. Tel est bien en tout cas ce que suggère l'idée kuhnienne voulant que les révolutions scientifiques finissent généralement par s'imposer à tous (Kuhn, 1983). Ainsi, les physiciens ont pu s'attacher longtemps aux idées d'espace absolu ou de détermi- 
nisme strict, mais leurs successeurs n'en ont pas moins fini par adopter la relativité et la mécanique quantique. Or, la question qui nous intéresse ici est celle de savoir s'il en va de même dans le cas de l'utilité marginale décroissante. Pour un économiste qui n'aurait retenu de l'apriorisme que le souci d'intelligibilité qui y est souvent associé, il ne saurait en aller tout à fait de même, car quelle que soit sa fécondité en termes de prédictions empiriques, une théorie basée sur des postulats faisant place à l'idée d'utilité marginale croissante ne pourrait être acceptée parce qu'une telle théorie rendrait inintelligible ce qu'il s'agit justement de comprendre.

Pour établir de façon convaincante aux yeux de ses opposants le bien-fondé d'une telle position, ou, si l'on préfère, pour établir cette position empiriquement, il faudrait donc pouvoir montrer que, mis en face d'une théorie qui invoquerait l'idée d'une utilité marginale croissante, mais qui serait plus féconde en prédictions que la théorie traditionnelle, les économistes opteraient néanmoins pour une théorie basée sur la notion d'utilité marginale décroissante. Mais comment réaliser une telle démonstration si de facto c'est bien cette théorie basée sur l'utilité marginale décroissante qui est la plus féconde? S'il est exact que cette théorie est la seule valable, on voit mal comment une théorie basée sur un postulat opposé pourrait parvenir à prédire un plus grand nombre de phénomènes. Puisqu'il ne semble guère possible qu'une telle théorie se présente à l'horizon, il y a bien peu de chances qu'on la voit un jour déboutée, malgré sa présumée supériorité en matière de prédictions, par une théorie basée sur la notion d'utilité marginale décroissante. Conséquemment, en l'absence d'une telle éventualité, on voit mal comment pourrait être mis en évidence, de façon un peu convaincante, le caractère incontournable de la préférence des économistes pour le principe d'utilité marginale décroissante. Si ce dernier principe est préféré à son présumé rival, les économistes pourront toujours alléguer que c'est en vertu de sa supériorité du point de vue empirique et non pas en vertu de sa présumée supériorité en tant que condition d'intelligibilité. Bref, le fait même d'avoir raison à propos de la théorie qu'il défend constituerait paradoxalement, pour notre économiste soucieux d'intelligibilité, un obstacle majeur à la possibilité d'établir empiriquement qu'il a raison de soutenir que les économistes adopteraient cette théorie même si elle se trouvait surpassée en matière de prédictions.

Toutefois, faute de pouvoir espérer établir empiriquement que la préférence accordée par les économistes à l'utilité marginale décroissante est d'une tout autre nature que celle que les physiciens ont accordée aux divers postulats théoriques qui se sont succédé au cours de l'histoire de la physique, il peut être intéressant d'illustrer ne serait-ce que l'enjeu d'une telle question. Puisque le problème auquel nous nous sommes heurtés est lié à l'absence de théories empiriquement fécondes qui seraient basées, par exemple, sur un principe d'utilité marginale croissante, et dont on pourrait établir qu'elles sont inacceptables parce qu'inintelligibles, on peut toujours 
imaginer un état de choses qui rendrait possible une telle théorie et voir ce qui s'ensuivrait ${ }^{7}$.

\section{Une expérience imaginaire: l'étrange cas des UMC}

Admettons donc, par hypothèse, qu'une enquête, menée avec toutes les garanties scientifiques requises, ait établi qu'un nombre appréciable d'êtres humains (appartenant à un groupe que j'appellerai UMC pour "utilité marginale croissante") adoptent systématiquement le comportement suivant. Supposons d'abord l'individu représentatif du groupe UMC confiné dans un lieu où il est totalement privé d'eau mais où il dispose d'une forte somme d'argent à laquelle il est fort attaché et admettons que, à toutes les heures, un marchand lui propose un litre d'eau en s'efforçant, chaque fois, d'obtenir de lui en retour la somme maximum qu'il peut lui arracher. Lors de la première offre d'eau, l'individu UMC refuse absolument de débourser plus de $10 \$$ pour obtenir un premier litre que, une fois acquis, il boira de manière à éviter la mort par déshydratation. Lors de la seconde offre cependant, et c'est ici qu'il se singularise comme membre du groupe UMC, il accepte de payer $25 \$$ pour le second litre avec lequel, sa soif maintenant apaisée, il s'empresse de se laver. Lors de la troisième offre, l'individu va même jusqu'à payer $100 \$$ pour acquérir un troisième litre que, cette fois, il consacrera à laver quelques objets de son entourage, après s'être assuré qu'il n'avait plus tellement soif et qu'il était lui-même suffisamment propre.

À certains égards, le comportement de cet individu paraît normal puisqu'il veille à satisfaire d'abord les besoins qu'il juge les plus urgents avant de porter attention à ceux qui lui paraissent moins importants. Toutefois, mesurée par ce qu'il est prêt à offrir pour acquérir successivement les diverses unités d'un bien, l'utilité marginale qu'il reconnaît à chaque litre qui lui est successivement offert croît de façon spectaculaire avec chaque nouvel achat. Moins est grande la priorité qu'il accorde par son comportement d'utilisateur aux besoins qu'il lui reste à satisfaire à l'aide d'un bien (dont, pourtant, on suppose ici que tous les usages possibles lui étaient connus par expérience), plus sont importantes les sommes qu'il est disposé à sacrifier en vue d'obtenir des unités supplémentaires de ce bien.

Un tel comportement pourrait nous paraître totalement irrationnel, mais ce serait en vertu d'une conception de la rationalité qui tiendrait à une sorte de reliquat d'apriorisme que chacun de nous continue peut-être à entretenir plus ou moins secrètement. Le comportement des membres du groupe UMC ne pourrait certes pas être qualifié de logiquement contradictoire puisqu'on pourrait aisément imaginer des robots qui fonctionneraient sans le moindre accroc en adoptant un comportement de

7. J'ai déjà eu l'occasion de proposer une version un peu moins élaborée de cette expérience imaginaire dans Lagueux, 1988. 
ce type. Ces robots seraient programmés de manière à offrir de plus en plus d'unités monétaires à mesure qu'on leur proposerait des unités supplémentaires d'un même bien qu'ils sauraient utiliser de manière à satisfaire en priorité les besoins les plus pressants, c'est-à-dire ceux, par exemple, dont la satisfaction contribuerait le plus à accroître leur efficacité. Pourquoi faudrait-il penser que l'"évaluation" qui les détermine à accorder tel ou tel montant à l'achat de telle ou telle unité du bien considéré doit être logiquement reliée à celle qui leur permet de comparer entre elles les "contributions à leur efficacité" de tel ou tel usage de ce bien? On serait tenté de répondre qu'il ne serait pas rationnel de séparer ces deux évaluations. Peut-être, mais ne serait-ce pas en vertu de quelque apriorisme larvé que nous invoquerions ainsi la structure de l'action rationnelle? D'un strict point de vue empiriste, le comportement de ces robots serait un fait parmi d'autres; on n'aurait donc pas à exiger que ce fait se conforme à la représentation que nous pouvons nous faire a priori de ce qu'est un comportement rationnel; on pourrait seulement noter que le comportement observé diffère singulièrement de celui des êtres humains considérés normaux.

Mais revenons aux membres du groupe UMC, car ce n'était que pour faire voir que le comportement de ces derniers ne correspondait nullement, en tant que tel, à une impossibilité logique qu'a été considéré ici le cas des robots. Par hypothèse, les membres du groupe UMC ne sont pas perçus comme des robots (lesquels seraient forcément programmés par des humains) mais bien comme des humains au comportement assez particulier. Néanmoins, comme dans le cas des robots, il y aurait lieu de considérer que ce comportement est un fait, même s'il diffère singulièrement de celui qu'il est habituel d'attribuer aux êtres humains. Quoi qu'il en soit, face à ce type de comportement que l'on supposera durable dans le temps, l'une ou l'autre de deux attitudes devrait finir par s'imposer, la première dictée par une logique d'inspiration aprioriste, la seconde par une logique d'inspiration empiriste.

(1) Un "aprioriste" refuserait d'accepter comme telle la possibilité même qu'il y ait utilité marginale croissante et se convaincrait qu'il faut absolument expliquer autrement le comportement des membres du groupe UMC, fût-ce à l'aide de considérations ad hoc. Par exemple, il pourrait supposer que les membres de ce groupe se réfèrent à quelque finalité cachée, qu'ils éprouvent un immense plaisir à méduser les marchands ou encore qu'ils ne possèdent pas vraiment certaines informations pertinentes ou qu'ils sont amenés, au cours même de l'expérience considérée, à modifier leurs goûts ou leur échelle de valeurs. À défaut de pouvoir retenir une explication de ce genre (ou de pouvoir en imaginer une plus astucieuse et plus satisfaisante), il ne lui resterait qu'à prétendre que ces individus sont dépourvus de raison, à moins qu'il n'en vienne, à la limite, à contester qu'ils soient d'authentiques êtres humains, tant il est convaincu a priori, que, pour être intelligible, une action humaine exige, en vertu de sa structure même, que l'utilité marginale soit conçue comme décroissante. 
(2) Bien sûr, un empiriste, quant à lui, ne serait pas tenu de rejeter illico l'idée voulant que l'utilité marginale soit décroissante ; il pourrait s'efforcer de conserver ce vieux paradigme qui l'avait bien servi jusqu'alors et chercher lui aussi à expliquer ce phénomène bizarre à l'aide de diverses hypothèses plus ou moins ad hoc. Seulement il ne saurait accorder à la notion d'utilité marginale décroissante un crédit tel qu'elle l'inciterait à faire prévaloir indéfiniment les solutions ad hoc et les interprétations tautologiques de l'utilité marginale décroissante contre des théories plus générales qui viseraient à rendre compte de l'ensemble des comportements observés. Une théorie générale de ce genre pourrait prendre appui, par exemple, sur une loi qui assurerait que, quand il s'agit d'obtenir des unités successives d'un même bien, la valeur absolue (ou le carré) de l'écart à la moyenne des prix qui seront effectivement payés décroît de façon continue, s'annule, puis crồt à nouveau jusqu'à épuisement des ressources. Une telle loi aurait indubitablement l'avantage de la généralité puisqu'elle s'appliquerait aussi bien aux individus qui se comportent de la façon traditionnelle qu'aux membres du groupe UMC. En effet, placés dans la même situation que celle qui a permis de prendre conscience de la singularité des membres du groupe UMC, les êtres humains considérés normaux paieraient d'abord beaucoup plus que la moyenne des sommes qu'ils seraient amenés à offrir au cours de l'expérience, mais cet écart diminuerait progressivement jusqu'à ce que, une fois le prix moyen atteint, la valeur absolue de cet écart à la moyenne se remette à crô̂tre, du fait qu'ils continueraient d'offrir de moins en moins pour chaque nouvelle unité. En cela, puisqu'il n'est question ici que de la valeur absolue de l'écart à la moyenne, ils obéiraient à la même loi que leurs congénères UMC. Comme on l'a vu, ces derniers paieraient d'abord beaucoup moins que la moyenne de ce qu'ils seraient ensuite amenés à offrir au cours de l'expérience, mais, dans leur cas aussi, la valeur absolue de cet écart à la moyenne diminuerait progressivement jusqu'à ce que, le prix moyen atteint, cet écart se remette à croître, puisqu'ils paieraient de plus en plus cher pour chaque nouvelle unité. Les graphiques suivants permettent de voir d'un seul coup d'œil que même si les membres du groupe UMC ont un comportement qui est en quelque sorte l'inverse de celui des êtres humains "normaux" (graphique 1), la façon dont varie la valeur absolue (ou le carré) de l'écart à la moyenne des sommes qu'ils sont respectivement prêts à verser (pour chaque unité successive) est identique dans les deux cas (graphique 2).

Il va sans dire que des économistes empiristes pourraient mettre au point des explications beaucoup plus sophistiquées que celle, quelque peu simpliste, qui est proposée ici. L'intérêt de cet exemple ne tient évidemment pas au fait qu'on pourrait le mettre en réserve en vue de l'utiliser dans l'éventualité où notre situation fictive se réaliserait, mais plutôt au fait qu'il permet de voir au nom de quoi "l'explication empiriste" proposée serait carrément rejetée par les économistes aprioristes ou cryptoaprioristes. À bien des égards, cette explication est pourtant plus satisfaisante que "l'explication aprioriste" puisqu'elle ne fait pas appel à des considérations ad hoc ou à des processus inobservables comme les changements de goûts. En outre, puis- 

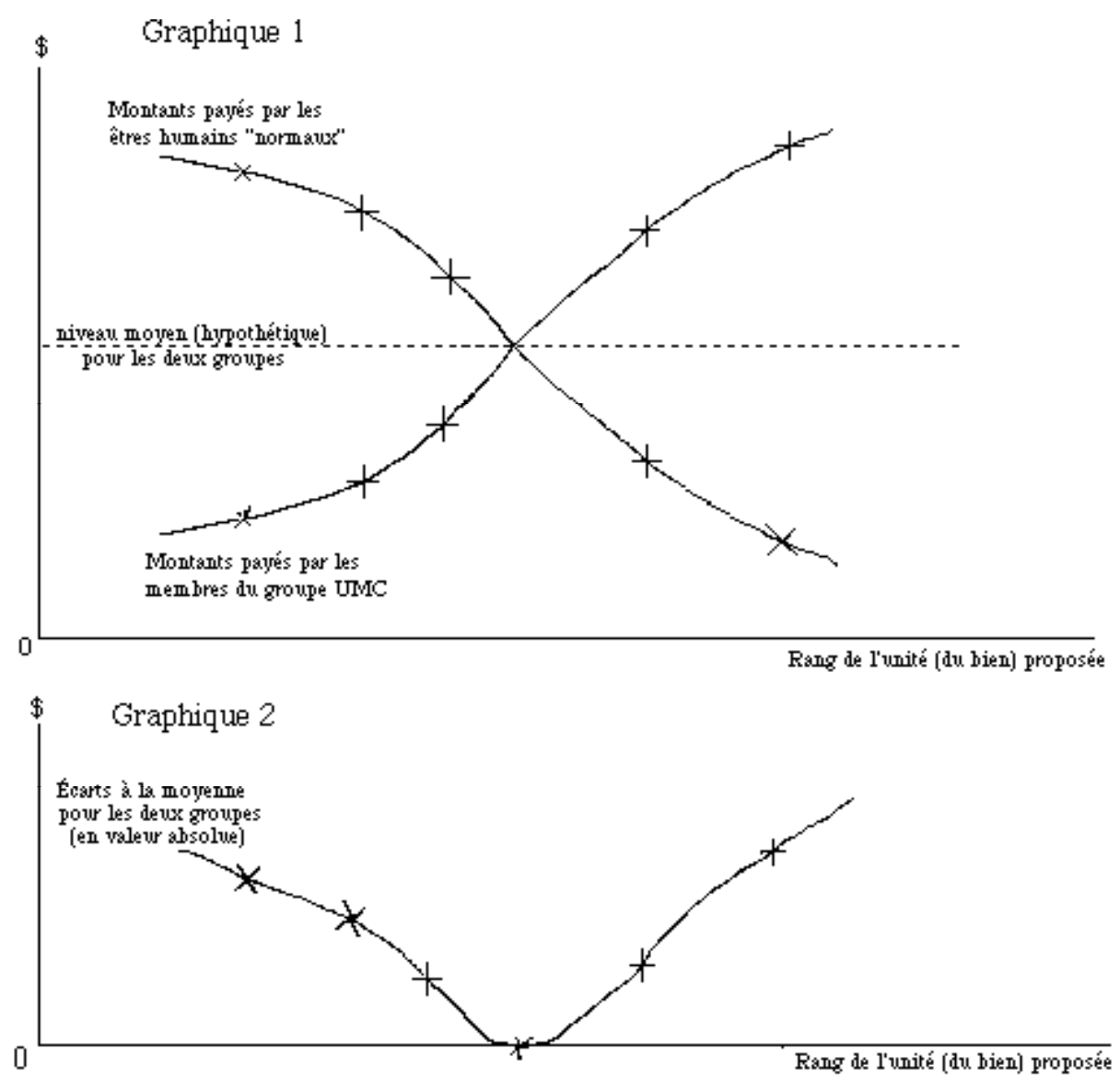

qu'elle s'applique indifféremment aux deux groupes d'individus, elle est plus générale et, de ce fait, plus élégante, et surtout elle surmonte, par hypothèse, l'épreuve des tests avec succès. Pourtant, il me paraît probable que la plupart des économistes estimeront qu'elle demeure totalement sans intérêt parce qu'elle ne permet nullement de comprendre le comportement des individus. Les oscillations, dans un sens ou dans l'autre, d'un écart (exprimé en valeur absolue) de part et d'autre d'une moyenne n'ont aucun sens du point de vue de l'action humaine alors que la décroissance de l'utilité marginale en a clairement un.

\section{Le statut épistémologique d'une condition d'intelligibilité}

Ce qui importe, ici, c'est de toucher du doigt l'enjeu réel du débat entre aprioristes et empiristes. Cet enjeu n'est pas vraiment, comme Mises le prétendait, de savoir s'il y a des lois connues strictement a priori qui seraient valables universellement. Ce ne peut même pas être de savoir si les économistes de facto refuseraient d'abandonner un principe comme celui de l'utilité marginale décroissante, 
car un tel débat ne reposerait que sur d'assez futiles supputations. Cet enjeu est plutôt celui de savoir si les économistes peuvent légitimement se donner pour objectif de rendre compte des actions humaines de manière telle qu'elles soient intelligibles.

Sans doute, ce parti pris en faveur de l'intelligibilité — qui est a priori, bien sûr, comme tout parti pris - ne récupère-t-il pas tout ce que l'on associe habituellement à l'apriorisme des Autrichiens, mais il rend compte d'un trait beaucoup plus fondamental de la pensée propre à ces économistes. Que certains membres de l'école autrichienne aient été amenés à voir dans l'existence de telles conditions d'intelligibilité un laissez-passer vers des connaissances a priori me paraît être un glissement malheureux mais explicable. Ce parti pris en faveur de l'intelligibilité n'autorise, en effet, en rien à parler de "lois a priori universellement valables". À vrai dire, la question de savoir, par exemple, si de facto l'utilité marginale est universellement décroissante n'est pas de celles auxquelles un économiste peut donner une réponse, même s'il est profondément convaincu de la nécessité de faire appel à la condition d'intelligibilité qui est associée à cette notion. Il s'agit plutôt d'une question oiseuse à laquelle on ne peut guère répondre de façon empirique, mais à propos de laquelle on ne saurait se contenter de réponses a priori. Cela toutefois importe peu, car tout ce qui peut être soutenu dans la perspective ici proposée, c'est qu'on ne voit pas comment on pourrait donner un sens à l'action humaine si l'on refuse d'admettre que, du moins en dernière analyse, l'utilité marginale doit être conçue comme décroissante.

Une condition d'intelligibilité de l'action humaine n'est pas davantage une hypothèse retenue parmi toutes celles qui pourraient contribuer à rendre compte des actions humaines. Elle est une condition sans laquelle l'action humaine devient tout simplement inexplicable au sens où l'expliquer ne voudrait plus rien dire. Pour parvenir à expliquer valablement le comportement des membres du groupe UMC, il faudrait, en effet, montrer que, compte tenu des désirs et des croyances - aussi bizarres qu'ils puissent paraître - de ces membres, cette condition d'intelligibilité, en dernière analyse, est bel et bien satisfaite. Si, par exemple, il était établi que, pour quelque raison, ces individus éprouvent à méduser les marchands d'eau une satisfaction supérieure à celle que leur procure l'usage de l'eau qu'ils achètent, il ne serait tout simplement plus vrai qu'ils sacrifient plus pour une satisfaction inférieure qu'ils le font pour s'offrir une satisfaction supérieure. Par contre, estimer que cette condition d'intelligibilité demeure insatisfaite, c'est estimer que ce comportement demeure inexpliqué ou, si l'on préfère, c'est estimer qu'il n'a littéralement aucun sens, ce qui, dans le cas des actions humaines, est équivalent à dire qu'il demeure sans explication.

C'est dans ce contexte que des économistes autrichiens ont été amenés à penser qu'il pourrait être plus important et plus fécond d'analyser les implications des actions humaines à la lumière de telles conditions d'intelligibilité que de chercher à respecter coûte que coûte les prescriptions de la méthode empirique. Ont-ils raison 
de le penser contre ceux qui préfèrent, du moins en principe, s'en tenir aux canons de l'empirisme? Il est bien difficile, rappelons-le, de répondre de façon concluante à une telle question. En fait, pour que l'on puisse trancher de manière décisive en faveur d'une interprétation empirique contre une interprétation basée sur cette condition d'intelligibilité, il faudrait qu'il soit possible de montrer empiriquement que le principe d'utilité marginale décroissante ne relève en rien d'une telle condition. Mais à quoi devrait ressembler cette démonstration? Il faudrait, par exemple, que l'on établisse que le comportement des membres du groupe UMC (en supposant contre toute attente qu'un tel groupe existe) demeure parfaitement intelligible même s'il contredit strictu senso le principe d'utilité marginale décroissante, ce qui, on l'a $\mathrm{vu}$, paraît difficilement concevable quand on a affaire à des actions humaines.

Il importe de rappeler ici qu'il ne suffit pas, pour y arriver, de prêter à ces individus le genre de défaillances (quant à leur information, à leur jugement, à leur volonté, etc.) dont l'existence nous permet de comprendre des comportements bizarres qui, à première vue, nous paraissent parfaitement stupides. Car admettre que, compte tenu de ces défaillances, on peut comprendre l'action de ces individus, c'est justement montrer que ces individus - à leur façon et compte tenu du contexte où ils se trouvent - ne violent pas le principe d'utilité marginale décroissante. En effet, pour peu que l'on admette que les membres du groupe UMC oublient ce que sont leurs besoins au moment où ils offrent une certaine somme, ou qu'ils ne connaissent pas vraiment la valeur de l'argent, ou qu'avec le temps ils deviennent de plus en plus incapables de refuser une offre - et de telles défaillances sont loin d'être inconcevables —, leur comportement devient intelligible, mais justement parce qu'il cesse alors de contredire le principe d'utilité marginale décroissante interprété strictu senso. On ne saurait non plus se contenter de soutenir que ces individus se comportent tout simplement comme le font les robots construits à leur modèle. En effet, on peut fort bien comprendre que des robots "se comportent" de la sorte, parce qu'ils auraient pu, par exemple, être programmés par un informaticien qui serait intéressé, pour des fins théoriques qui me semblent parfaitement compréhensibles, à illustrer à l'aide de la cybernétique ce à quoi pourrait ressembler un comportement contredisant le principe d'utilité marginale décroissante. Mais on ne se résoudrait quand même pas à invoquer les intentions d'un Programmeur de l'histoire humaine pour rendre intelligible les actions humaines attribuées aux UMC. Manifestement, la seule façon d'expliquer de telles actions tout en s'épargnant la peine de prêter à tout prix un sens à des comportements qui semblent en être dépourvus serait de montrer que ceux-ci se comprennent - un peu comme la chute des corps se comprend - parce que, compte tenu des circonstances, ils peuvent être dérivés d'une loi générale (mais pas forcément intelligible en tant que telle) comme le serait, si toutefois elle pouvait être établie sur d'autres bases, la "loi des écarts à la moyenne" évoquée plus haut.

En somme, pour qu'une telle "loi générale" ait une quelconque valeur explicative, il faudrait qu'elle soit logiquement reliée - comme le sont les lois de la gravi- 
tation et de la chute des corps - à un corpus théorique permettant de dériver un ensemble impressionnant d'autres phénomènes également établis empiriquement. Il faudrait, par exemple, que l'on en vienne à trouver qu'il est tout à fait normal pour un individu de veiller jalousement à ce que l'écart (en termes absolus) entre la somme qu'il est prêt à payer et la moyenne (de toutes les sommes qu'il paiera et qu'il a déjà payées pour un bien donné) diminue progressivement jusqu'à atteindre zéro de manière à se mettre ensuite à croître. Bref, il faudrait que, dans ce contexte, il nous paraisse parfaitement acceptable que les membres du groupe UMC s'entêtent à offrir plus pour satisfaire un besoin jugé moins important que ce qu'ils offrent pour satisfaire un besoin jugé plus important, pourvu que, ce faisant, ils respectent une loi des écarts à la moyenne aux applications si étendues qu'il serait déraisonnable de la rejeter! La question est de savoir si la satisfaction d'une loi d'apparence aussi farfelue que celle des écarts à la moyenne peut avoir, pour le théoricien, une valeur explicative qui l'amènerait à conclure qu'un comportement est expliqué avec son aide. Pour cela, il faudrait que le caractère "normal" d'un tel comportement dérive tout naturellement de sa conformité à une sorte de loi de la nature qui aurait le mérite d'avoir été établie empiriquement et de s'intégrer de manière cohérente au corpus d'une science empirique.

Or, compte tenu des résultats empiriques encore assez limités auxquels ont donné lieu les sciences humaines, l'idée même que l'on puisse un jour dériver les choix et les actions des êtres humains de théories empiriques de ce type paraît relever de la science-fiction. Dans ce contexte, rejeter les explications fort éclairantes qui sont fondées sur des motivations rationnelles dans l'espoir de parvenir à expliquer les actions humaines à partir de théories aussi hypothétiques consiste à rien moins qu'à abandonner la proie pour l'ombre. Dans ces conditions, il n'est pas étonnant que la plupart des économistes aient été amenés à traiter, au moins implicitement, le principe d'utilité marginale décroissante (ou, pour des raisons analogues, la décroissance du taux marginal de substitution) comme une condition d'intelligibilité plutôt que comme une hypothèse empirique susceptible d'être remplacée par une autre. Sans doute, ne peut-on pour autant qualifier d'aprioristes ces économistes dont la grande majorité, au demeurant, ne voient pas là une raison de renoncer à leurs convictions empiristes. On peut toutefois penser qu'ils adoptent cette position parce qu'ils perçoivent plus ou moins confusément qu'il n'est guère raisonnable, pour les raisons explicitées plus haut, d'attendre d'une quelconque enquête empirique des résultats susceptibles de débouter de façon décisive les relents d'"apriorisme" qu'on pourrait associer à une telle condition d'intelligibilité. D'ailleurs, ils sont d'autant moins enclins à rejeter le principe d'utilité marginale décroissante que l'abandon du mode d'explication fondé sur des motivations rationnelles (mode d'explication qui présuppose ce principe) impliquerait la mise au rancart de l'objet de la science économique telle qu'on la comprend depuis son origine, puisqu'un comportement qui serait régi par une loi mécanique comme celle des écarts à la moyenne ne serait plus, à proprement parler, un comportement économique. 
On le voit, ces principes économiques auxquels on peut ainsi être conduit à attribuer une validité a priori ne sont guère autre chose que des applications du principe de rationalité qu'on ne saurait établir empiriquement mais qui n'en constitue pas moins une condition d'intelligibilité incontournable pour quiconque entend rendre compte des actions humaines. Reste à se demander si les économistes qui adoptent cette façon de voir ne font pas appel à un mode de connaissance épistémologiquement fort suspect en tenant pour acquis, sans le démontrer vraiment, que le principe de rationalité - et certains de ses dérivés comme le principe d'utilité marginale décroissante - constituent une telle condition d'intelligibilité? On peut toutefois écarter aisément l'essentiel de cette difficulté en faisant observer qu'ils n'ont tout simplement aucun besoin d'établir la validité de ce principe et de ses dérivés, puisqu'on a affaire aux principes mêmes qui sont présupposés par la plupart des questions auxquelles ils s'affairent, par leurs explications, à apporter des réponses. C'est justement parce que le principe de rationalité ou ses dérivés paraissent violés qu'une explication économique devient nécessaire. Considérons quelques exemples. Comment se fait-il qu'en certaines circonstances la demande d'un produit s'accroît alors même que son prix monte? Comment le prix d'une marchandise peutil se fixer à un niveau qui ne semble correspondre en rien aux intérêts et aux visées des individus concernés? Comment se fait-il qu'une baisse générale des salaires n'entraîne pas une réduction du taux de chômage? À ces demandes d'explication, l'économiste répondra en montrant que malgré les apparences, le principe de rationalité et ses dérivés ne sont pas vraiment violés. La plupart du temps, en effet, il s'agira pour lui de montrer qu'en analysant bien les situations en cause, on constate que les comportements présumés anormaux s'expliquent parfaitement si l'on suppose que les individus sont guidés par ce qu'ils estiment être leur meilleur intérêt.

Il est évident qu'aucun économiste ne peut établir a priori que les êtres humains se comportent de façon rationnelle, ou que les fonctions d'utilité marginale et de demande (laquelle, comme on sait, est étroitement liée à la précédente) doivent être décroissantes. Toutefois, indépendamment du fait qu'il se dise aprioriste ou empiriste, un économiste qui est confronté, par exemple, à une hausse de la demande des pommes de terre consécutive à une hausse de leur prix (et donc à une situation paradoxale en ce qu'elle pourrait laisser penser que la fonction de demande des pommes de terre n'est pas décroissante) est confronté à un problème réel justement parce qu'il voit mal comment il pourrait être rationnel pour des individus de se comporter de façon aussi bizarre. Face à cette situation, il pourrait se contenter de rappeler que puisque rien ne prouve que les individus doivent être rationnels, il n'y a pas de problème à ce que la fonction de demande prenne une forme en apparence aussi inattendue. En effet, si une sorte d'ultra-empirisme l'incitait à renoncer allègrement à l'idée voulant qu'une fonction de demande, tout comme une fonction d'utilité, doive en principe être décroissante et, plus généralement, à renoncer au principe de rationalité, cet économiste ne verrait pas pourquoi une forme convient mieux qu'une autre à une fonction de demande et ne verrait donc pas qu'il y a là une énigme à résoudre. Pour avoir mis en question, au nom de cet ultra-empirisme, les 
conditions mêmes de la vie économique en même temps que les postulats qui leur sont associés, cet économiste risquerait fort de ne plus guère rencontrer de problèmes proprement économiques auxquels il pourrait apporter une solution typiquement économique.

Par contre, si, à l'instar des économistes qui ont résolu le paradoxe posé par le type de bien qui est associé au nom de Robert Giffen, cet économiste estime qu'il y a bien là un problème proprement économique, c'est parce qu'il voit quelque chose d'intellectuellement gênant à ce que la situation décrite se produise dans un univers composé d'individus présumés rationnels. Si cet économiste, faisant sienne la solution standard proposée en réponse à ce problème, rappelle que pour qui tient compte de l'effet de revenu, et non seulement de l'effet de substitution, la hausse de la demande des pommes de terre devient parfaitement compatible tant avec un comportement rationnel qu'avec une augmentation de leur prix, on ne saurait contester qu'il apporte une solution à un problème bel et bien réel. Il n'est pas pour autant en mesure d'établir que les individus sont rationnels ou que la courbe de demande des pommes de terre est décroissante, mais il peut néanmoins affirmer que ces principes sont des conditions d'intelligibilité de l'action humaine, dans la mesure où ce n'est que si on les admet qu'il y a des problèmes proprement économiques engendrés par des actions humaines et, partant, des solutions à ces problèmes.

Or, à cet économiste, on ne saurait reprocher de faire appel à des principes qui n'ont pu être établis empiriquement puisque les principes qu'il invoque sont ceux-là mêmes (rationalité du comportement, utilité marginale décroissante, etc.) qui font qu'il y avait un problème à résoudre. L'économiste qui les invoque comme éléments de solution ne saurait être accusé de les prendre indûment pour acquis, puisque ces principes ne constituent rien de moins qu'une des données du problème qu'il veut résoudre, lequel tient essentiellement au caractère inintelligible d'une situation où ces principes ne seraient pas respectés. Si, par contre, on refuse de reconnaître la moindre validité à ces principes, le problème auquel Giffen voulait répondre cesse tout simplement d'en être un. Sans doute un économiste pourrait-il se fixer pour objectif à long terme d'expliquer pourquoi, en cas de hausse des prix, la demande de pommes de terre décroît en certaines occasions et s'accroît en d'autres et de le faire sans recourir au principe de rationalité et donc sans rien présumer quant à l'évolution normale de l'utilité marginale et de la demande. Il n'en reste pas moins que, même s'il parvenait à le faire de façon satisfaisante, ceci n'enlèverait rien au fait que l'énigme de Giffen se trouve légitimement expliquée par les économistes qui invoquent ce principe et ses dérivés. Si la plupart des économistes autrichiens ont préféré s'en tenir à la discussion des conséquences de principes de ce genre, dans lesquels ils voyaient une condition d'intelligibilité, plutôt que de s'engager dans une analyse strictement empirique qui ne respecterait pas plus cette condition d'intelligibilité que ne le faisait la "loi" sur l'évolution des écarts à la moyenne, c'est qu'ils estimaient plus important, sur le plan scientifique, de contribuer à l'intelligibilité d'un problème que de poursuivre une démarche qui, à cause de sa fidélité aveu- 
gle aux canons de l'empirisme, aurait risqué de leur faire rater l'occasion d'une telle contribution.

Tel est, à mon sens, l'essentiel de ce qui peut subsister de "l'apriorisme" avec lequel les économistes autrichiens se sont, à des degrés divers, reconnu certaines affinités. Si l'on accepte la présente interprétation, cet "apriorisme" s'oppose à l'empirisme, non pas par la réponse qu'il apporterait à la question de savoir d'où nous viennent nos connaissances, mais par celle qu'il apporte à la question de savoir ce que présuppose le fait de prétendre rendre compte des actions humaines et des phénomènes économiques. 


\section{BIBLIOGRAPHIE}

Alter, Max, 1990, "What do we know about Menger?", dans Caldwell, pp. 313-48. Barrotta, Pierluigi, 1996, "A Neo-Kantian Critique of Von Mises's Epistemology", Economics and Philosophy, 12, pp. 51-66.

Barrotta, Pierluigi, 1998, "Parsons on Mises and Kant: a Comment", Economics and Philosophy, 14, pp. 127-30.

Caldwell, B., 1984, "Praxeology and its Critics", History of Political Economy, 16, pp. 363-79.

Caldwell, B. (dir.), 1990, Carl Menger and his Legacy in Economics (supplément annuel de History of Political Economy, no 22), Durham, Duke University Press.

Dolan, E.G., 1976, The Foundations of Modern Austrian Economics, Kansas City, Sheed and Ward.

Friedman, Milton, 1953, "The Methodology of Positive Economics", pp. 3-43, in Friedman, Milton, 1953, Essays in Positive Economics, University of Chicago Press, Chicago.

Grassl Wolfgang et Barry Smith, 1986, Austrian Economics, Historical and Philosophical Background, Londres et Sydney, Croom Helm.

Hicks, J.R. et Allen, R.G.D., 1934, "A Reconsideration of the Theory of Value", Partie I (par J.R. Hicks), Economica New series 1, p. 52-76.

Hutchison, Terence W., 1956, "Professor Machlup on Verification in Economics", Southern Economic Journal, 22, pp. 476-83.

Kaufmann, Felix, 1937, "Do Synthetic Propositions a Priori Exist in Economics, A reply to Dr. Bernardelli", Economica, 4, pp. 337-42.

Kirzner, Israel, 1976, "On the Method of Austrian Economics" in Dolan, pp. 40-51.

Klant, Johannes J., 1984, The Rules of the Game, The Logical Structure of Economic Theories, Cambridge, Cambridge University Press (première édition en néerlandais, 1973 comme thèse doctorale, puis 1979).

Kuhn, Thomas S., 1983, La Structure des révolutions scientifiques, 2ème éd., (traduit de l'anglais par Laure Meyer), Paris, Flammarion (première édition en anglais, 1962).

Lachmann, Ludwig M., 1969, "Methodological Individualism and the Market Economy" in Streissler et alii, 1969, pp. 89-103.

Lagueux, Maurice, 1988, "Apriorisme et empirisme en science économique", Fundamenta Scientiae, 9, p. 217-30.

Lagueux, Maurice, 2004,"The Forgotten Role of the Rationality Principle in Economics", Journal of Economic Methodology, 11(1):31-51.

Meidinger, Claude, 1978, "Apriorisme et théorie économique", Revue économique, 2, pp. 261-90. 
Mises, Ludwig von, 1944, "The Treatment of 'Irrationality' in the Social Sciences", Philosophy and Phenomenological Research, 4, 3, Juin.

Mises, Ludwig von, 1976 Epistemological Problems of Economics, New York, New York University Press.

Mises, Ludwig von, 1978, The Ultimate Foundation of Economic Science, Kansas City, Sheed Andrews and McMeel (première édition: 1962)

Mises, Ludwig von, 1985, L'Action humaine (traduit de l'américain par Raoul Audouin), Paris P.U.F. (première édition américaine, 1949).

Mises, Ludwig von, 1985a, Theory and History, Auburn, Alabama et Washington, D.C., Auburn University Press (première édition, 1957).

Parsons, Stephen D., 1990, "The philosophical Roots of Modern Austrian Economics: Past Problems and Future Prospects", History of Political Economy, 22(2):295-319.

Parsons, Stephen D., 1997, "Mises, the A Priori, and the Foundations of Economics: a Qualified Defence", Economics and Philosophy, 13, pp. 175-96.

Rothbard, Murray, N, 1957, "In defense of 'Extreme Apriorism'", Southern Economic Journal, 23, pp. 314-20.

Rothbard, Murray, N., 1962, Man, Economy and State: A Treatise on Economic Principles, Princeton, N.J., D. Van Nostrand, 19, vol. I.

Rotwein, Eugene, 1986, "Flirting with Apriorism: Caldwell on Mises", History of Political Economy, 18, pp 669-673, réplique à Caldwell, 1984.

Smith, Barry, 1986, "Austrian Economics and Austrian Philosophy", in Grassl \& Smith, pp. 1-36.

Smith, Barry, 1996, "In defense of Extreme (Fallibilistic) Apriorism", Journal of Libertarian Studies, 12, pp. 179-92.

Streissler, Erich, et alii (dir.), 1969, Roads to Freedom, Londres, Routledge and Kegan Paul. 